\title{
Verzeichnis der bis jetzt erschienenen Nummern.
}

1. Otto, Traucrspiel v. $F, M, K$ linger. Her. v. B. Seuffert. VII, $108 \mathrm{~S}$. 1881. $90 \mathrm{Pf}$.

2. Voltaire am Abend seiner Apotheose, v. H. I. Wagner. Her. v. B. Seuffert. XI, 19S.1881.40 Pf.

3. Fausts Leben vom Maler Müller. Her. v. B. Se uffert.XXVI, 116S. 1881.

M. 1.10 .

4. Preuss. Kriegslieder v. e.Grenadier von $J . W .1$. Gleim. Her.v. A. Sa uer.XXXVII, 44S.1882.70Pf.

5. Faust, ein Fragm. v.Goelhe. Her.v. B.Seuffert. XV,89S.1852.80Pf.

6. Helmannv.O.M.Wieland.Her.v.F. Muncker.XXX,116S.1882.M.1.20

7. 8. Frankfurt.gelelnte Anzeigen vom Jalue 1772. (Lingeleitet von Wilh. Scherer, her. v. B. Seuffert.) ErsteHälfte.S.1-352.1882. M. 2.80. Zweite Hälfte nebst Einleitung u. Register. S. 353-700 u. CXXIX S.1883.M.3.80,zus.M.6.60

9. Karl v.Burgund,e.Trauersp.(nach Aeschylus) v. J.J. Bodmer. Her. v. B.Seuffert. XII, 26S. 1883.50Pf.

10. Yersuch einiger Gedichte von F. v. Hagedom. Her. von Aug. Sauer. XI, 99 S. 1883. 90 Pf.

11. DerMessias,erster,zweiter u.dritt. Gesang v, F'G.Klopstock. Her.v.F. Muncker.XXXI,84S.1883.90Pf.

12. Vicr kritische Gellichte v. $J . J$. Bodmer. Her. v. J ak. Bächtold. XLVII, 110 S. $1883 . \quad$ M. 1.20.

13. Die Kindermörderim, e.Trauerspiel v. H. L. Wagner nebst Szenen aus den Bearbeitungen K. G.Lessings u. Wagners. Her.v. Erich Schmidt. X, 116 S. 1883. M. 1.-

14. Ephemerides und Volkslieder v. Goethe. Her.von Ernst Martin. $\mathrm{XX}, 47$ S. 1883.

$60 \mathrm{Pf}$.

15. GustarWasa v.C.Brentano.Her.v. J.Min or.XIV,136S.1883.M.1.20.

16. De la littérature allemande von Friedrich d. Gr. Her. v. Lu d wig Geiger.XXX, 37 S.1883. 60 Pf.

17.18.19.A.W.Schlegels Vorlesungen iber schïne Litteratur u. Kunst 1884. Her. v.J ak. M in or. Erster Teil:DieKunstlelure.LXXII,370S. M. 3.50. Zweiter Teil: Gesch. der klass. Litt. XXXII, 396S. M. 3.50. Dritter Teil: Gesch. d.romant.Litt. (nebst Personenreg.z.d. 3 Teilen). XXXLX,252S.M.2.50, zus.M.9.50. 20. Gedanken uber dieNachalumung der griech. Werke in derMalerei u.Bildhauerkunst $v . J . J . W i n c k e l$ mann. Erste Ausgabe 1755 mit Oeser's Vignetten. Eingeleitet von Ludw.v.Urlichs, her.v.B.Seuffert. X, 44 S. 188 o. $70 \mathrm{Pf}$.

21. Die guten Franen v. Goethe. Mit Nachbildg.d.Originalkupfer.Her.v. B.Seuffert.XI,27 S. 1885. 70Pf.

22. Freundschaftl.Lieder v.J.J.Pyra und S. G. Lange. Her. v, A u gu st Sauer. L, 167 S. 1885. M. 1.80.

23. Anton Reiser, ein psycliol.Roman v. K. Ph.Moritz. Her.v.L.Geiger. XXXVIII, 443 S. 1886 . M. 3.80.

24. Ueber meine theatral.Laufbahn v. $A . W . I f f l a n d . H e r . v . H$ u g o Holstein. CVI, 130 S. 1886. M. 2.-

25. KleineSchriften z.Iunst v.Heinr. Mever.Her.v.Paul Weizs äcker. CLXIX, 258 S. 1886. M. 4.20.

26. Joh. Elias Schlerels ästhetische und dramaturgische Schriften. Her. von Joh. v. Antoniewicz. CLXXX, 226 S. 1887. M.4.-

27. HeinrichHeinesBuch derLieder nebst ein. Nachlese nach den ersten Druck. od.Handschr. Her.v.Ernst Elster. CLIV,255S.1887.M. 4.-

28. Die Mätresse. Lustspiel von $K$. G. Lessing. Her.v. Eugen Wolff. XX, 113 S. 1887.

M. 1.30 .

29. 30. Briefe iib. Merkwürdigkeiten der Litteratur. Her.v.A lex.v on Veilen.29. Frste u.zweite Samml. S. 1-1S7. 1858.M.1.80.30. Dritte Sammlung nobst Einleitg.CXLIX, S. 188-367.1889.ML.4, zus.M.5.80.

31. Ueber d. bill. Nachalunung des Schöncn v. K.Ph, Movitz. Her.v.S. A uerbach.XIV,45S.1885.90Pf.

32. Julias von Tarent $u$, die dramatisclien Fragmente v. J.A. Leisewitz. Her. v. Rich. M. Werner. LXVIII, 143 S. 1889. M.2.$33-38 . \mathrm{Uz}$, sämtl poetischeWerke. Her, von A. Sauer, CIX, $422 \mathrm{~S}$. 1890.

M. 8.40 .

39. Das Fanstbuch des Christlich Meynenden. Nach dem Druck von 1725 her.v.Siegfr.Szamatólski. Mit 3 Portr. XXVI, 30S. 1891. M.1.60.

Alle Nummern anch gebunden. Preis des Einbandes, braua Lelawand mit Rotschnitt, 80 Pf. 


\section{Deutsche Litteraturdenkmale d. 18. u. 19. Jahrh.}

40-41. Von dentsch. Art u. Kunst. Einige flieg. Blätter. Her. v. H. Lambel. 1892. XVII, $124 \mathrm{~S}$. M. 3.50.

42. Joh. Nicolaus Götz, Gedichte. Her. von C. Schüddekopf 1893. XXXVI, 89 S. M. 2.40.

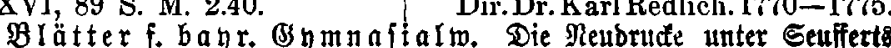

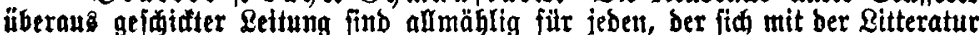

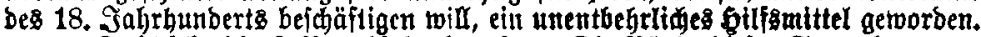

Beitidrift f. $\mathfrak{R} \mathfrak{c} \mathfrak{a} \mathfrak{l}$ f

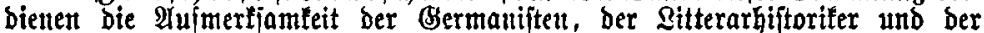

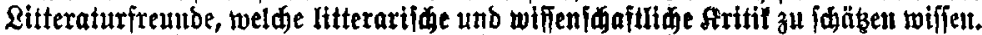

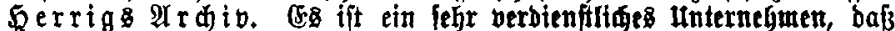

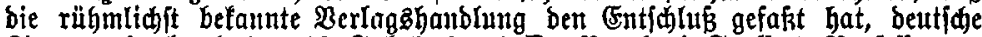
Eitteraturbentmale bes 18. Jahrh. Durd Dr. Bernhard Senffert, \$rofeffor an ber llutiberfität Braz, in Reubrưfen heraugageben za laffett.

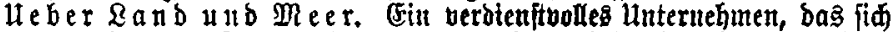

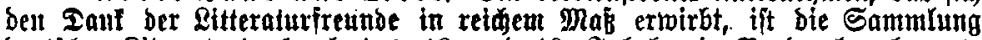

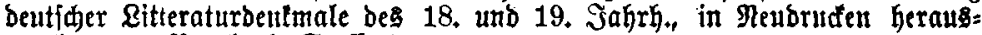
gegeben von Bernharb Seuffert.

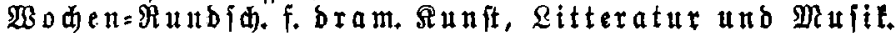
Eg jei ber außserorbentliđe $\mathfrak{B e r t}$ ber jebent Ritteraturhiftorifer utb = Freunbe foft unentbchrltâen "Deutjđent Qitteraturbenfntale" wärmitens betont. Die Sanumlung, in ber witroigften und jaditiften 1 Beife bon berufenen Belehrten ergänż mı erweitert, gereiđt biejen, twie ber unermübliđ auf eine ben ftrengiten

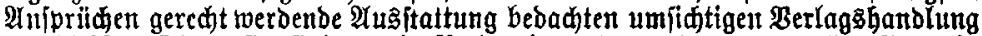

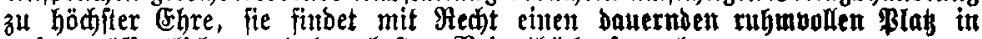
unieren öffentlid)en unb ben bejten Sribatbïberiammlunget.

\section{Kleine Bibliothek}

zur

\section{deutschen Litteraturgeschichte.}

je in elegantem Leinwandband 80 Pf.

Bdch. I a. Althochdentsche Litteratur mit Grammatik, Uebersetzung und Erläuterungen von Prof. Th. Schauffler.

Sammlung Göschen Nr. 28.

\# Ib. Nibelungen and Kudran in Ausw. und Mittelhochdeutsche Grammatik mit kurzem Wörterbuch von Dr. W. Golther. 2. Aufl. 1892.

Sammlung Göschen Nr. 10.

7

II. Hartmann ron Ane, Wolfram von Eschenbach und Gottfried von Strassburg. Auswahl aus dem höf. Epos mit Anmerkungen und Wörterbuch von Dr. K. Marold.

Sammlung Göschen Nr. 22.

" III. Walther ron der Vogelweide mit Auswahl aus Minnesang und Spruchdichtang mit Anmerkungen und Wörterbuch v. Prof. O. Güntter.

Sammlung Göschen Nr. 23.

"IV. Seb. Brant, Hans Sachs, Luther, Fischart, mit Auswahl von Dichtungen des 16. Jahrh. mit Anmerkungen von Dr. L. Pariser. Sammlung Göschen Nr. 24.

V. Kirchenlied und Volkslied. Geistliche und weltl. Lyrik des 17. u. 18. Jabrh. bis auf Klopstock. Mit Anmerkungen von Dr. G. Ellinger. Sammlung Göschen Nr. 25. Hieran reihen sich Sammlung Göschen Nr. 1-9, 21 etc. 